\title{
Liver transplantation in adult polycystic liver disease: the Ontario experience
}

\author{
Mohammed Alsager ${ }^{1}$, Shuet Fong Neong ${ }^{2}$, Radhika Gandhi ${ }^{1}$, Anouar Teriaky ${ }^{1}$, Ephraim Tang ${ }^{2}$, \\ Anton Skaro², Karim Qumosani ${ }^{1}$, Les Lilly², Zita Galvin², Nazia Selzner ${ }^{2}$, Mamatha Pallavi Bhat ${ }^{2}$, Klajdi Puka ${ }^{3}$ \\ and Mayur Brahmania ${ }^{1,4^{*}}$
}

\begin{abstract}
Background: Liver transplantation (LT) remains the curative treatment for symptomatic Polycystic Liver Disease (PCLD) patients and is associated with excellent survival rates. The aim of the study is to review the Ontario experience in LT for PCLD.

Methods: A retrospective study was performed from pre-existing LT databases from the LT Units at Toronto General Hospital and London Health Sciences Center, which are the two LT programs in Ontario, Canada. This database contains demographic, clinical parameters and follow-up of all patients transplanted for PCLD. Data was extracted for patients who underwent LT between January 2000-April 2017 and included follow up until December 31st, 2018.

Results: A total of 3560 patients underwent LT, of whom 51 (1.4\%) had PCLD and met inclusion criteria. 43 (84\%) of these patients were female. The median physiologic Model for End Stage Liver Disease (MELD-Na) score at time of referral was 13 (IQR $=7-22)$, however all patients required MELD-Na exception points to receive LT. The median age of transplant was 62 years (IQR $=59-64)$ for male vs. 52 (IQR $=45-56)$ for female patients. 33 (65\%) of our cohort had PCLD while 9 (17.5\%) had ADPKD and 9 (17.5\%) had both diseases. 39 (76\%) had LT due to symptoms of mass effect, while $8(16 \%)$ had portal hypertensive complications. After a median follow-up of 6.3 (IQR $=2.9-12.5)$ years, the probability of survival was $96 \%$ (95\% Cl: 90\%, 100\%). Log-rank test, comparing survival analysis between males and females did not show a statistically significant difference $(p=0.26)$.
\end{abstract}

Conclusion: Most patients underwent LT for PCLD due to symptoms of mass effect with women being more likely than men to undergo LT. LT for PCLD had excellent long-term survival.

Keywords: Polycystic liver disease, Liver transplantation, PCLD outcome

\section{Background}

Polycystic liver disease (PCLD) can occur in isolation or, more commonly, as an extra-renal manifestation of autosomal dominant adult polycystic kidney disease (ADPKD). For the group of patients that develop only renal cysts at diagnosis, $30 \%$ can develop liver

\footnotetext{
*Correspondence: mbrahmania@gmail.com

1 Division of Gastroenterology and Multi-Organ Transplant, Western University, London, ON, Canada

Full list of author information is available at the end of the article
}

cysts within 30 years of diagnosis and the prevalence is estimated to be 1:5000 [1-3]. In patients with ADPKD, PCLD is diagnosed when there are more than 20 cysts present and more than $94 \%$ of those aged 35 years and above will have hepatic cysts as the size increases with age (1-3\%/year) [4]. Other risk factors associated with severe cystic disease in ADPKD include female gender, exogenous female hormones, multiple pregnancies and a greater burden of renal cysts.

As hepatic cysts enlarge, they cause a mass effect and exert pressure on adjacent organs. As a result, patients 
experience dyspnea, early satiety, weight loss and malnutrition [4]. Portal hypertension can also arise as large hepatic cysts cause architectural distortion, increasing intrahepatic resistance to blood flow. Hepatic venous outflow obstruction and direct portal vein compression also contribute to the mechanism of portal hypertension in this patient group [4]. Liver transplantation (LT) is the definitive curative treatment for PCLD patients with excellent patient survival rates when compared to patients transplanted for other indications. The indication for Liver transplant (LT) is usually for symptomatic relief. Once listed, Patients with PCLD are awarded exceptions MELD-Na points every three months. In this study, we examined the Ontario experience with regards to LT in PCLD patients.

\section{Methods}

\section{Study design, inclusion and exclusion criteria}

A multi-center retrospective cohort study was performed of patients diagnosed with PCLD and referred for LT between January 1, 2000 and April 30, 2017. The study was conducted at the two LT centers in the province of Ontario (University Health Network, Toronto; and University Hospital; London) which serve an estimated population of 14,000,000 and perform approximately 225 LT per year (including living donor and simultaneous liver kidney (SLK)). The diagnosis of PCLD was based on radiographic evidence (CT or MRI). Patients who were transplanted (single organ, SLK, or living donor) for complications related to PCLD were included in the study. Patients were excluded from LT if there had any contraindications such as poor social support, frailty or uncontrollable psychiatric illness. All data was extracted for patients who had follow up till December 31st, 2018. We also excluded patients whom follow-up data is unavailable in the electronic health records. Figure 1 shows the final patients selection. The study was conducted in accordance with the guidelines of the Declaration of Helsinki and the principles of Good Clinical Practice and is approved by the ethical review boards of University of Toronto and Western University.

\section{Statistical analysis}

Continuous variables were expressed as median values with interquartile ranges (IQR) and categorical variables were expressed as frequencies (percentages). Continuous variables were compared between groups using the Wilcoxon rank-sum test and categorical variables were compared using Fisher's exact test. The starting time point, or baseline, for the analysis was defined as the date a patient received LT. Patients were followed until death or date of the last outpatient visit. Data was analyzed using the $R$ statistical software (Version 3.2.2).

\section{Results}

\section{Patient characteristics}

There was a total of 3560 LT performed during the study period with 51 (1.4\%) related to PCLD and meeting inclusion criteria. 43 (84.3\%) LT recipients were female, while $8(15.6 \%)$ recipients were male. The median age for all patients was 54 years $(\mathrm{IQR}=46-59)$. In subgroup analysis, the median age for female recipients was 52 years $(\mathrm{IQR}=45-56)$ vs. a median age of 62 years in male recipients $(\mathrm{IQR}=59-64)$. The median waitlist time was 9.7 months (IQR $=5.5-18.1)$. The median physiologic MELD score at the time of transplant was 13 (IQR =7-22). The median physiologic MELD score for male population was $22(\mathrm{IQR}=17-22)$ in comparison to a median physiologic MELD of 10 (IQR $=6-21)$. In the male population, 7 (87\%) had worsening kidney function with a median creatinine of $426(\mathrm{IQR}=237-$ $485)$ as oppose to female group $15(34.8 \%)$ which had a median creatinine of 91 with (IQR $=73-184)$. However, all patients were transplanted with exception points. 39 (76\%) were referred for LT, as they were experiencing mass effect symptoms such as early satiety, abdominal pain, nausea and vomiting and 8 (16\%) had portal hypertension related complications as the main indication for LT. The remaining $4(8 \%)$ patients' indications of transplant were not documented. In patients with portal hypertension, $5(62.5 \%)$ had portal hypertension due to vascular invasion while the remaining 3 (37.5\%) had portal hypertension due to fibrotic parenchyma. All patients had registered dietitian involved in their as part of transplant team however, none of the patients required additional nutritional support i.e., TPN. Table 1 show the demographic characteristics of our cohort.

\section{Main disease and type of LT}

33 (64.7\%) patients had PCLD as the primary disease, 9 (17.6\%) had polycystic kidney disease (PCKD) as their primary disease and $9(17.6 \%)$ patients had both polycystic liver and kidney disease. 29 (56.9\%) received LT alone, 7 (13.7\%) had a simultaneous liver kidney transplant (SLK) and 15 (29.4\%) patients received a kidney transplant followed by LT. There were 46 (90\%) orthotopic LT and 5 (10\%) living donor LT. Out of patients who received orthotropic LT, 38 (82.6\%) were female. All patients who received living donor $\mathrm{LT}$ were females.

\section{Post-operative management, complications and survival}

Patients were followed up for a median of 6.3 years $(\mathrm{IQR}=2.9$-12.5), however, male patients were followed up for a median of 2.9 years (IQR $=2.2-4.1$ ) vs a median of 8 years (IQR $=3-13.8)$ among females. At the end of follow-up, 16 (31.4\%) were using Tacrolimus monotherapy while a combination of Tacrolimus and 


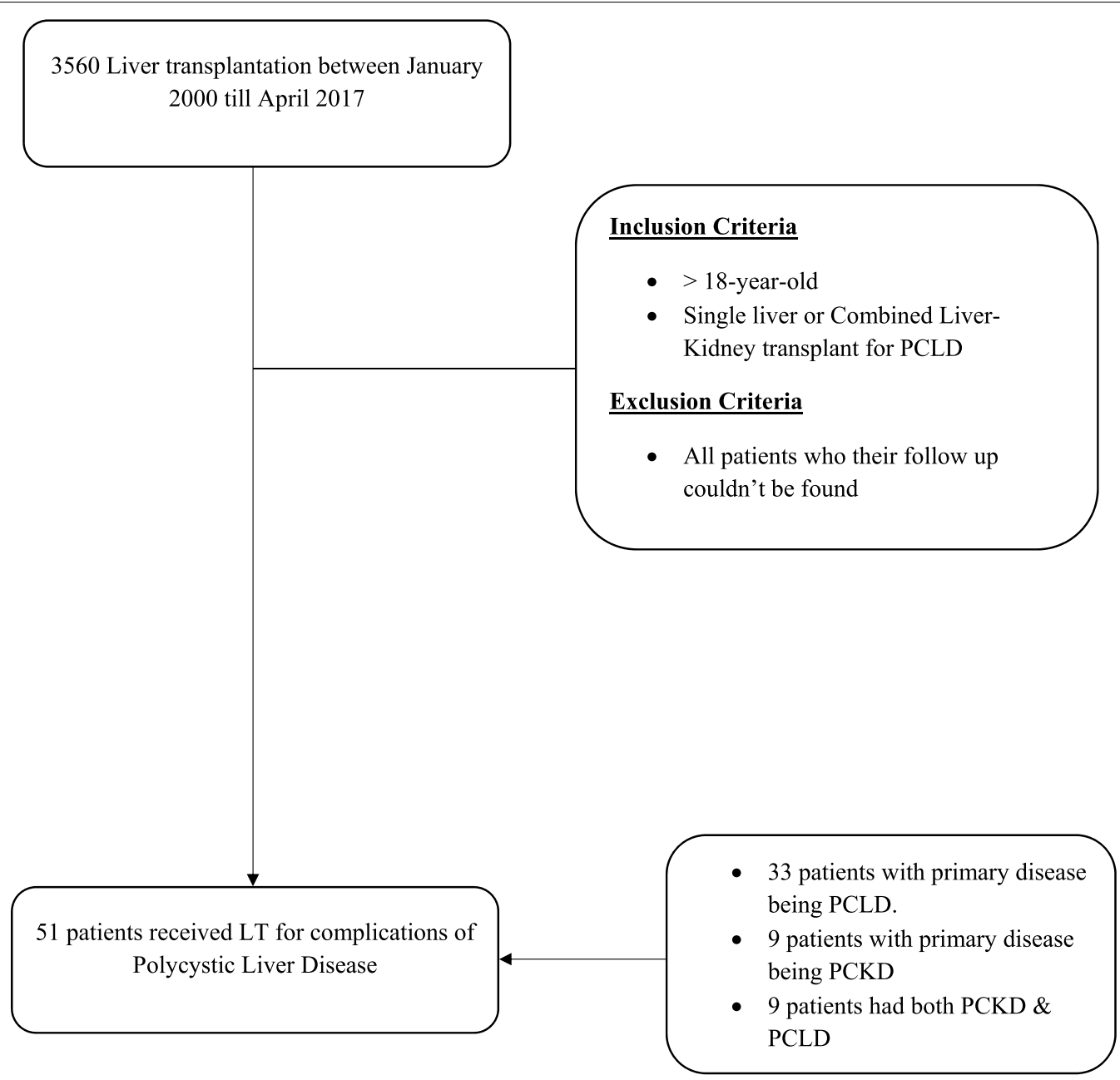

Fig. 1 Patient selection

Mycophenolic acid was used in 11 (21.5\%) patients. 24 (47\%) patients were on combination therapy of Cyclosporin/ Mycophenolic or Cyclosporine alone. 1 (2\%) of the patients developed insulin dependent Diabetes Mellitus, 2 (3.9\%) developed CMV infection, 3 (5.8\%) developed complications related the surgery such as pneumothorax/ pleural effusion. In addition, 3 (5.8\%) developed T-cell mediated rejection and 2 (3.9\%) had re-transplantation due hepatic artery thrombosis. There were 4 total deaths in our cohort, all greater than one year from transplant and were transplantrelated ( 1 male, 3 females) with failed grafts. Log-rank test comparing survival among males and females was not significant $\left(\chi^{2}(1)=1.3, p=0.26\right.$; Fig. 2$)$. The overall probability of survival for our cohort is $100 \%$ (95\% CI $100 \%, 100 \%)$ at 1 year and $96 \%(95 \%$ CI 90\%, 100\%) at the $3,5 \& 10$ year follow up.

\section{Discussion}

Our study shows patients with PCLD undergo LT predominantly for symptom management, more specifically for the mass effect caused by the multiple hepatic cysts. Native MELD scores tend to be much lower than what would usually be needed to attract a deceased donor offer and therefore most attract a donor with the help of additional MELD exception points. Although most PCLD LT recipients were female, both genders had 5-year survival exceeding 90\%.

PCLD is a disease characterized by presence of multiple cysts in the liver and is often associated with adult polycystic kidney disease (ADPKD). Isolated PCLD is usually associated with gene SEC63 and PRKCXH, which code for special proteins involved in protein processing [5]. Once mutated, these proteins affect polycystin-1 and polycystin-2 which will lead the formation of cysts [6]. 
Table 1 Patient demographics of those undergoing liver transplantation for polycystic liver disease

\begin{tabular}{|c|c|c|c|}
\hline Variable & Total & Male & Female \\
\hline Number of Transplants & $51(100 \%)$ & $8(16 \%)$ & $43(84 \%)$ \\
\hline Median age* & $54(46-59)$ & $62(59-64)$ & $52(45-56)$ \\
\hline Median Waitlist (months) & $9.8(5.5-18.7)$ & $10.2(7.4-14.1)$ & $9.5(5-18.8)$ \\
\hline Median MELD score at time of transplant & $13(7-22)$ & $22(17-22)$ & $10(6-21)$ \\
\hline Median (years) follow up & $6.3(12.5)$ & $2.9(2.2-4.1)$ & $8(3-13.8)$ \\
\hline Orthotopic Liver transplant & $46(90.1 \%)$ & $8(15.6 \%)$ & $38(74.5 \%)$ \\
\hline Living donor & $5(9.8 \%)$ & $0(0 \%)$ & $5(9.8 \%)$ \\
\hline Liver transplant alone & $29(56.8 \%)$ & $1(1.9 \%)$ & $28(54.9 \%)$ \\
\hline Kidney first then liver transplant & $15(29.4 \%)$ & $3(5.8 \%)$ & $12(23.5 \%)$ \\
\hline Simultaneous Liver-Kidney transplant (SLK) & $7(13.7 \%)$ & $4(7.8 \%)$ & $3(5.8 \%)$ \\
\hline Median Creatinine ( $\mu$ mol) & $101(80-319)$ & $426(237-485)$ & $91(73-184)$ \\
\hline Median Hemoglobin (g/L) & 116(109-126) & $118(113-127)$ & $116(109-127)$ \\
\hline Median platelet $\left(10^{9} / \mathrm{L}\right)^{*}$ & $189(130-239)$ & $189(118-203)$ & $193(132-240)$ \\
\hline Median Bilirubin $\mu$ mol* & $10(8-14)$ & $10(9-11)$ & $10(7-14)$ \\
\hline Median INR* & $1.1(1-1.2)$ & $1.2(1.15-1.2)$ & $1.1(1-1.2)$ \\
\hline Median Albumin $(\mathrm{g} / \mathrm{L})^{*}$ & $40(35-43)$ & $36(33-40)$ & $40(35-43)$ \\
\hline Death & $4(7.8 \%)$ & $1(1.9 \%)$ & $3(5.8 \%)$ \\
\hline
\end{tabular}

* Medians expressed as IQR
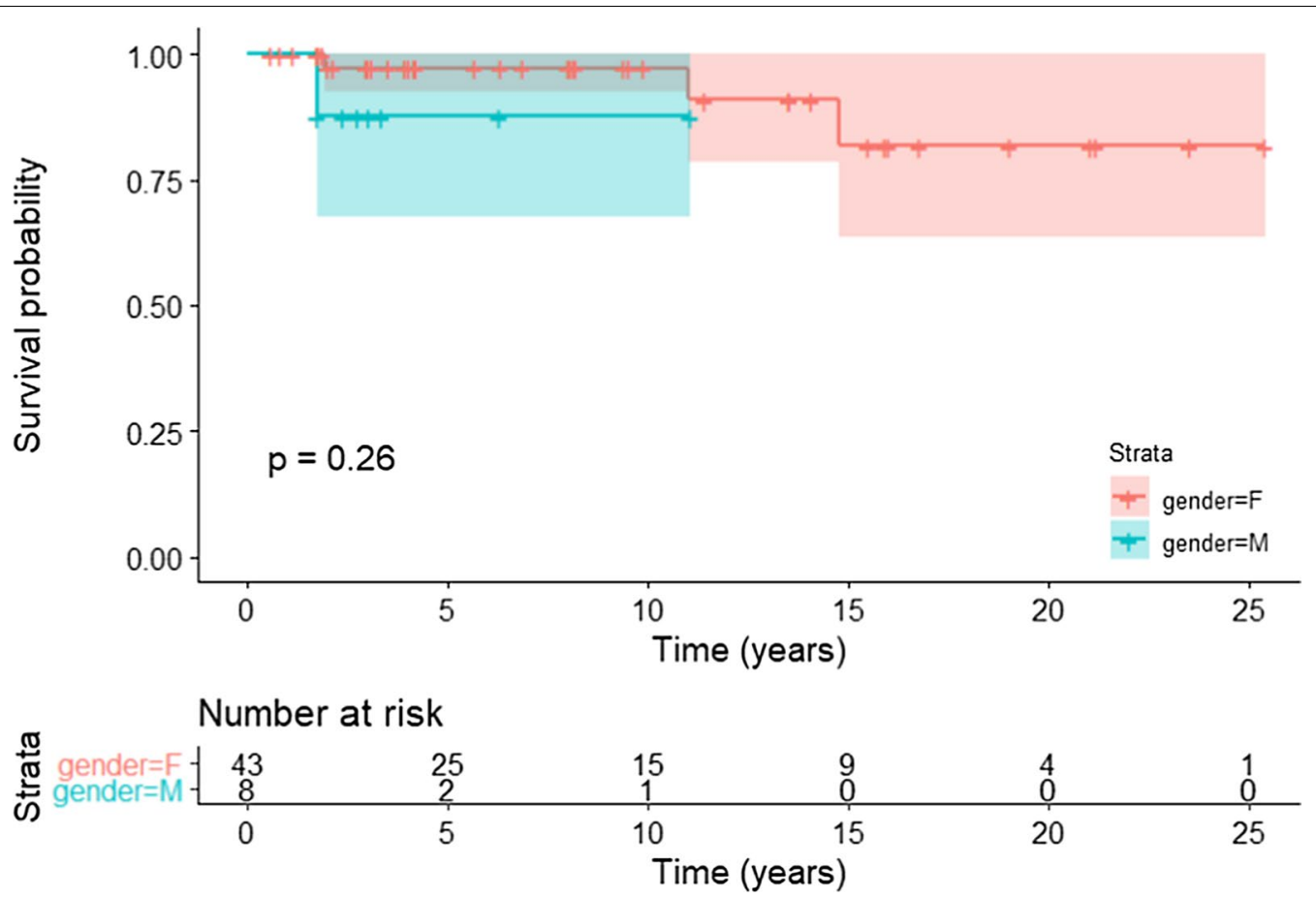

Fig. 2 Kaplan Meier curve showing survival analysis post-transplant between male and female over time (years)

The main risk factors for having PCLD is having a family member with the disease, as it follows Mendelian mode of inheritance. Despite the similar genetic predisposition, the major risk factors for a massive hepatic cyst are female sex, exposure to female steroid hormone, pregnancy and exogenous hormone use such as oral contraceptives (OCPs) in postmenopausal women. This finding has led to the theory of probable hormonal regulation of these proteins leading to formation of hepatic cysts. This likely also explains why majority of PCLD patients 
are female, as estrogen stimulates cystolic receptors through a complicated signaling pathway leading to cyst proliferation [7].

There are multiple risk factors that play a pivotal role in predicting mortality in LT. In general, these factors are related to the pre-transplant kidney function, cardiac \& pulmonary status [8]. Nonetheless, most LT programs have allocated exception points for PCLD patients taking this into account when it comes to prioritizing PCLD patients in LT waitlist [9]. Our study had a median age of 54 years which may have contributed to the excellent outcomes shown when compared to the European liver transplant registry group (ELTR) who had 1, 5, 10 -year survival $89 \%, 85 \%, 77 \%$, respectively, for patient with PCLD [10]. This finding may be related to having a relatively younger population that were otherwise healthy and potentially have contributed to the excellent outcomes seen for our population. However, there are modalities that can be tried before proceeding with LT.

Generally, patients with PCLD receive LT after exhausting medical therapy such as somatostatin analogues, radiological therapies such as sclerotherapy, cyst fenestration and lastly liver resection [11]. Despite the short acting half-life of naturally occurring somatostatin, synthetic long-acting somatostatins have been developed. Somatostatin interferes with biochemical signaling pathway inhibiting fluid secretion and have shown excellent results in patients with PCLD [12]. However, side effects may not be preferable to patients since it causes diarrhea and abdominal cramps. In the past, it has been suggested that combination of mammalian target of rapamycin (mTOR) inhibitors with somatostatin analogues may have better outcomes in comparison to somatostatin analogues monotherapy, but a recent trial suggested otherwise [13]. Aspiration with sclerotherapy is another treatment modality which considered safe and has regression and partial regression of $22 \%$ and $19 \%$ respectively [14]. With regards to cyst fenestration, patients' needs to be selected carefully as it is preferred in patients with less severe disease and few, superficial, dominant hepatic cysts [15]. Lastly, patients may undergo hepatic resection with preservation of liver volume, however, this does not come without potential complications [16]. These complications can vary from anastomotic leak to massive ascites and should be considered in highly selected patients in which immunosuppressant is not desired. Therefore, it is reasonable to consider medical therapy with somatostatin analogues for patients with diffuse PCLD and to consider radiological and surgical treatments for patients with limited but large cysts before assessing for LT [17].

Liver transplantation has become a lifesaving procedure for many patients who meet criteria, however, this is limited by shortage of organ donors which makes organ allocation challenging for patients who need it the most [18]. PCLD patients often do not show the typical cirrhosis pathway sequelae from compensation to decompensation but rather have indications for LT due to symptom burden (cachexia, anorexia, abdominal distention) and to improve their quality of life. This leads to an ethical dilemma whether we subject otherwise healthy patients to lifelong immunosuppression only to improve their quality of life [19]. In addition to the above-mentioned challenges, this has led to increased wait times and waitlist mortality among other populations including patients with PCLD [21]. Recently, a similar situation was thought to be occurring with Hepatocellular carcinoma, and hence, the LT programs in Ontario decided to cap exception points at 30 and this may be an avenue to explore with PCLD given treatment modalities available and the excellent outcomes [22].

The strength of this study includes a large sample size from two established LT program that covers a population of 14 million people. However, there are important limitations of the current study that need to be highlighted. Firstly, the possibility of selection bias as PCLD patients are usually healthier compared to other patients with CLD may overestimate their survival. Also, our study population consists of mostly female patients limiting the generalizability to male patients, however, the nature of this disease dictates that it predominantly affects females. Secondly, no/missing data were available regarding surgical techniques, transfusion requirements, post-operative renal replacement therapy requirements, radiological and histopathological findings thus it was not included in our analysis. Lastly, our study included patients who had LT from 2000-2017 in two LT centers that have different surgical and therapeutic protocols which also may have affected survival however, surgical techniques and protocols have improved over time and so it is likely outcomes would at least be similar or even better.

\section{Conclusion}

Patients with PCLD carry a high symptom burden requiring MELD-Na exception points to receive liver transplantation as many do not have high MELD-Na scores at the time of listing. Moreover, PCLD disproportionately affects women but overall long-term survival is excellent in both genders.

\footnotetext{
Abbreviations

LT: Liver Transplant; PCLD: Polycystic Liver Disease; MELD-Na: Model of End Stage Liver Disease-Na; IQR: Interquartile Range; ADPKD: Adult Polycystic Kidney Disease; SLK: Simultaneous Liver-Kidney; CT: Computed Tomography; MRI: Magnetic Resonance Imaging; PCKD: Polycystic Kidney Disease; CMV:
} 
Cytomegalovirus; ELTR: European Liver Transplant Registry; CLD: Chronic Liver Disease; mTOR: Mammalian target of rapamycin.

\section{Acknowledgements}

Not applicable.

\section{Authors' contributions}

Study concept and design were done by MA, LL, SN, MB. Statistical analysis and interpretation of data were done by MA and MB. MA, SN, LL, MB helped Drafting the manuscript. All authors read and approved the final manuscript.

\section{Funding}

None.

\section{Availability of data and materials}

Datasets used and/or analyzed during the current study are available from the corresponding author on reasonable request.

\section{Declarations}

Ethics approval and consent to participate

The study was approved by Western University Health Science Research Ethics Board (HSREB). Reference number: 111153.

\section{Consent for publication}

Not applicable.

\section{Competing interests}

The authors declare that they have no competing interests.

\section{Author details}

${ }^{1}$ Division of Gastroenterology and Multi-Organ Transplant, Western University, London, ON, Canada. ${ }^{2}$ Multi-Organ Transplant Program, University Health Network, Toronto, ON, Canada. ${ }^{3}$ Department of Epidemiology and Biostatistics, Western University, London, ON, Canada. ${ }^{4}$ Room A10-224; London Health Sciences Centre: University Hospital, Western University, London, ON N6A 5A5, Canada.

Received: 13 November 2020 Accepted: 28 February 2021

Published online: 09 March 2021

\section{References}

1. Berrebi G, Erickson RP, Marks BW. Autosomal dominant polycystic liver disease: a second family. Clin Genet. 2008;21(5):342-7. https://doi. org/10.1111/j.1399-0004.1982.tb01381.x.

2. Qian Q. Isolated Polycystic Liver Disease. Adv Chronic Kidney Dis. 2010;17(2):181-9. https://doi.org/10.1053/j.ackd.2009.12.005.

3. Chandok N. Polycystic liver disease: a clinical review. Ann Hepatol. 2012;11(6):819-26. https://doi.org/10.1016/s1665-2681(19)31406-1.

4. Mikolajczyk AE, Te HS, Chapman AB. Gastrointestinal manifestations of autosomal-dominant polycystic kidney disease. Clin Gastroenterol Hepatol. 2017;15(1):17-24. https://doi.org/10.1016/j.cgh.2016.06.017.

5. van Aerts RMM, van de Laarschot LFM, Banales JM, Drenth JPH. Clinical management of polycystic liver disease. J Hepatol. 2018;68(4):827-37. https://doi.org/10.1016/j.jhep.2017.11.024.

6. Rockey DC. Advances in hepatology: 2018. Curr Opin Gastroenterol. 2018;34(3):121-2. https://doi.org/10.1097/MOG.0000000000000438.

7. Everson GT, Helmke SM. Somatostatin, estrogen, and polycystic liver disease. Gastroenterology. 2013;145(2):279-82. https://doi.org/10.1053/j. gastro.2013.06.025.
8. Adam R, Cailliez V, Majno P, et al. Normalised intrinsic mortality risk in liver transplantation: European Liver Transplant Registry study. Lancet. 2000;356(9230):621-7. https://doi.org/10.1016/S0140-6736(00)02603-9.

9. US Department of Health and Human Services. Guidance to Liver Transplant Programs and the National Liver Review Board for: Adult MELD Exception Review. US Department of Health and Human Services website. Published online 2017. https://optnpilot.unos.org/media/2847/liver _guidance_adult_meld_201706.pdf

10. Adam R, Karam $\vee$, Delvart $\vee$, et al. Evolution of indications and results of liver transplantation in Europe. A report from the European Liver Transplant Registry (ELTR). J Hepatol. 2012;57(3):675-88. https://doi. org/10.1016/j.jhep.2012.04.015.

11. Cnossen WR, Drenth JPH. Polycystic liver disease: an overview of pathogenesis, clinical manifestations and management. Orphanet J Rare Dis. 2014;9(1):1-13. https://doi.org/10.1186/1750-1172-9-69.

12. Gevers TJ, Drenth JP. Somatostatin analogues for treatment of polycystic liver disease. Curr Opin Gastroenterol. 2011;27(3):294-300. https://doi. org/10.1097/MOG.0b013e328343433f.

13. Chrispijn M, Gevers TJG, Hol JC, Monshouwer R, Dekker HM, Drenth JPH. Everolimus does not further reduce polycystic liver volume when added to long acting octreotide: results from a randomized controlled trial. J Hepatol. 2013;59(1):153-9. https://doi.org/10.1016/j.jhep.2013.03.004

14. Drenth JPH, Chrispijn M, Nagorney DM, Kamath PS, Torres VE. Medical and surgical treatment options for polycystic liver disease1. Hepatology. 2010;52(6):2223-30. https://doi.org/10.1002/hep.24036.

15. Schnelldorfer T, Torres VE, Zakaria S, Rosen CB, Nagorney DM. Polycystic liver disease: a critical appraisal of hepatic resection, cyst fenestration, and liver transplantation. Ann Surg. 2009;250(1):112-8. https://doi. org/10.1097/SLA.0b013e3181ad83dc.

16. Aussilhou B, Douflé $G$, Hubert $C$, et al. Extended liver resection for polycystic liver disease can challenge liver transplantation. Ann Surg. 2010;252(5):735-41. https://doi.org/10.1097/SLA.0b013e3181fb8dc4.

17. Temmerman F, Missiaen L, Bammens B, et al. Systematic review: the pathophysiology and management of polycystic liver disease. Aliment Pharmacol Ther. 2011;34(7):702-13. https://doi.org/10.111 1/j.1365-2036.2011.04783.x.

18. Model M, Ahearn A. Ethical dilemmas in liver transplant organ allocation: is it time for a new mathematical model? AMA J Ethics. 2016;18(2):12632. https://doi.org/10.1001/journalofethics.2016.18.2.nlit1-1602.

19. Doshi SD, Bittermann T, Schiano TD, Seth D. Likely to be transplanted than those with chronic liver failure. Transplantation. 2018;101(8):1838-44. https://doi.org/10.1097/TP.0000000000001711.Waitlisted.

20. van Keimpema L, Nevens F, Adam R, et al. Excellent survival after liver transplantation for isolated polycystic liver disease: an European Liver Transplant Registry study. Transpl Int. 2011;24(12):1239-45. https://doi.org /10.1111/j.1432-2277.2011.01360.x.

21. Segev DL, Kucirka LM, Oberai PC, et al. Age and comorbidities are effect modifiers of gender disparities in renal transplantation. J Am Soc Nephrol. 2009;20(3):621-8. https://doi.org/10.1681/ASN.2008060591.

22. Brahmania M, Marquez V, Kneteman NM, et al. Canadian liver transplant allocation for hepatocellular carcinoma. J Hepatol. 2019;71(5):1058-60. https://doi.org/10.1016/j.jhep.2019.07.016.

\section{Publisher's Note}

Springer Nature remains neutral with regard to jurisdictional claims in published maps and institutional affiliations. 